\title{
Cognitive Function and Quality of Life in Egyptian Children with Acute Lymphoblastic Leukemia
}

\author{
Yahia $\mathbf{S}^{1}$, El-Hadidy MA ${ }^{2}$, El-Gilany $\mathbf{A H}^{3}$, Mansour AK ${ }^{1}$, Abdelmabood $\mathbf{S}^{1}$, Al wakeel AA ${ }^{1}$ and Darwish $\mathbf{A}^{1^{*}}$ \\ ${ }^{1}$ Department of Pediatric, Faculty of Medicine, Mansoura University, Egypt \\ ${ }^{2}$ Department of Psychiatry, Faculty of Medicine, Mansoura University, Egypt \\ ${ }^{3}$ Department of Public Health, Faculty of Medicine, Mansoura University, Egypt
}

*Corresponding author: Dr. Ahmad Darwish, Lecturer of pediatric hematology, oncology and bone marrow transplantation, Department of Pediatric, Mansoura University Children`s Hospital, Mansoura University, Mansoura-35516, Egypt, Tel: 00201001179115; E-mail: ahmad_darwish_77@yahoo.com

Received date: May 29, 2015, Accepted date: July 02, 2015, Publication date: July 06, 2015

Copyright: (c) 2015 Yahia S, et al. This is an open-access article distributed under the terms of the Creative Commons Attribution License, which permits unrestricted use, distribution, and reproduction in any medium, provided the original author and source are credited.

\begin{abstract}
Background and Objectives: Leukemic children face many obstacles that interfere with social activities and are at greater risk for neurocognitive dysfunction. This study aimed at highlighting the problems affecting quality of life in leukemic children given chemotherapy without irradiation.
\end{abstract}

Methods: A Cross-sectional comparative study was conducted in 177 leukemic patients, with 281 subjects as a healthy control. All patients and control were subjected to thorough history and examination. Cognitive functions were examined using the Mini-Mental State Examination. Paediatric quality of life was measured using paediatric quality of life inventory version 4.

Results: Health and activity, emotion, social relationship and school achievement were significantly impaired among leukemic children. Orientation, registration, attention, recall and language were adversely affected in leukemic children in comparison to healthy children.

Conclusion: Neurocognitive function and quality of life in leukemic children treated with chemotherapy were significantly impaired as compared with the healthy group.

Keywords: Leukemia; Children; Cognitive function; Quality of life; Egypt

\section{Introduction}

Similar to children suffering from chronic diseases, leukemic patients face many obstacles that interfere with their academic achievement and social activities. This is in part attributed to frequent school absenteeism, feeling of loneliness and the worry of school personnel about dealing with them [1]. Moreover leukemic children are at greater risk for neurocognitive dysfunction owing to treatment regimens used in childhood acute leukaemia. Both chemotherapy particularly methotrexate and craniospinal irradiation are generally associated with poor neurocognitive outcome [2].

The functional neurocognitive domains that are adversely affected by cancer treatments are attention, executive functioning, processing speed, working memory, and ability to learn, which impair the academic performance and social relationship of childhood cancer survivors [3]. Different mechanisms tried to explain these neurological and intellectual disabilities however damage involving the cortical and subcortical white matter is the most highlighted one [4-6].

Establishment of delicate balance between effective cancer therapy and the lowest morbidity remains a real challenge among oncologists. As adoption of more intensive treatment strategy even though better cure rate may be achieved, it can lead to unacceptable toxicity [2].
Focusing upon effects of chemotherapy alone during childhood acute lymphoblastic leukemia (ALL) treatment on neurocognitive function as well as quality of life (QOL) did not receive the same researchers' interest as the craniospinal irradiation did. In this study we aimed at highlighting the neurocognitive deficits and problems affecting the quality of life (QOL) in children treated with ALL chemotherapy alone without using craniospinal irradiation.

\section{Patients and Methods}

This study is a cross-sectional comparative study that was carried out in Pediatric Oncology Unit - Oncology Center Mansoura University during the period from $1^{\text {st }}$ December, 2013 to $30^{\text {th }}$ November, 2014. All children (198) of both sexes, aged from 9 to 15 years old with ALL who received chemotherapy without craniospinal irradiation for childhood ALL for at least 6 months or those who finished chemotherapy and followed at the study locality were enrolled. Patients exposed to craniospinal irradiation, suffering from mental deficits (IQ below 70), medical or psychiatric disorders were excluded. The comparison group included 295 children from a school near Mansoura University Hospital (from $3^{\text {rd }}$ Primary school class till 3rd Preparatory school) with age range from 9-15 years old. The comparison group was selected through systematic random sampling method from the list of students.

All children (both ALL patients and survivors as well as comparison group) were interviewed using Mini International Neuropsychiatric Interview for children MINI-KID Arabic version $[7,8]$ to exclude any 
children with psychiatric disorders from studied sample. In the group of patients and survivors, 9 children refused to participate in this study and 8 others were excluded from the study due to their psychiatric disorders (6 major depressive disorders and 2 children obsessivecompulsive disorders). Further, 4 other children were excluded due to their inability to complete the study tools. Therefore, the final total number of patients and survivors who actually participated in the study was 177 from the initial 198. In the healthy group, 13 children refused to participate in this study and another one child could not understand study tools, and they were excluded from this study. Therefore, the final total number of the healthy control group was 281 from the initial 295.

Data was using a special designed sheet to include sociodemographic data (age, sex, residence, consanguinity, birth order); treatment factors (treatment compliance, frequency of hospital admission, frequency of follow up in the outpatient clinic).

Cognitive functions were examined using the Mini-Mental State Examination (MMSE) $[9,10]$ and the trail-making test (Part $\mathrm{A}$ and Part B) which is a quick and easily administered test of visuo-motor tracking, conceptualization, and mental set shifting was used $[11,12]$.

Pediatric quality of life was measured using pediatric quality of life inventory version 4 (PedsQL 4.0) [13] which is Child self-report measured in children ages 5-18 years. Generic Core Scales have been used internationally and translated into many languages including Arabic. There are 4 subscales of the PedsQL 4.0 Generic Core Scales: Physical functioning ( 8 items), Emotional functioning (5 items), Social functioning (5 items), and School functioning ( 5 items).

Study protocol was approved by Medical Research Ethics Committee of Mansoura University-Faculty of Medicine. Written consent from the parents and their children were obtained at the beginning of the assessment.
Data was analyzed using SPSS version 16. Qualitative variables were presented as number and percent. Chi square test was used for comparison between groups. Quantitative variables were tested for normality distribution by Kolomogrov-Smironov test. Normally distributed variables were presented as mean (SD) and unpaired t-test was used for comparison between groups. Non-parametric variables were presented as median (min-max) and Mann-Whitney test was used for comparison between groups. $\mathrm{P} \leq 0.05$ was considered statistically significant.

\section{Results}

The socio-demographic data were demonstrated in Table 1 . No statistical significance was noted as regards to age, sex, residency, consanguinity or birth order between both groups.

Quality of life components (health and activity, emotion, social relationship with others and school achievement) all were significantly impaired among ALL cases when compared to their healthy peers (The median for health and activity problems was 450 vs. 800 , for emotion was 325 vs. 500, for social relationship was 350 vs. 500 and for school achievement was 200 vs. 500 respectively [p $\leq 0.001$ ] (Table 2). It is worth mentioning that some patients gave a score of zero as regard health and activity problems, emotion and school achievement according to the PedsQL 4.0 Generic Core Scales [13]. Similarly, cognitive function; measured by MMSE (orientation, registration, attention, recall, language and total MMSE) and Tail making tests (A $\&$ B) were adversely affected in ALL children in comparison to healthy children with statistically significant results ( $p \leq 0.001$ for orientation, attention, recall, language and total MMSE while $\mathrm{p}=0.039$ for registration) (Table 3).

\begin{tabular}{|c|c|c|c|c|}
\hline & & $\begin{array}{l}\text { Cases (177) } \\
\mathrm{N}(\%)\end{array}$ & $\begin{array}{l}\text { Comparison group (281) } \\
\mathrm{N}(\%)\end{array}$ & Sign. test \\
\hline \multicolumn{2}{|l|}{ Age $(X \pm S D)$} & $11.9 \pm 1.5$ & $11.96 \pm 1.6$ & $\mathrm{t}=0.13, \mathrm{P}=0.9$ \\
\hline \multirow[t]{2}{*}{ Sex } & M & $114(64.5)$ & $169(60.1)$ & \multirow[t]{2}{*}{$X^{2}=1.1, P=0.3$} \\
\hline & $\mathrm{F}$ & $63(35.5)$ & 112 (39.9) & \\
\hline \multirow[t]{2}{*}{ Residence } & $\mathrm{R}$ & $156(87.9)$ & $243(86.5)$ & \multirow[t]{2}{*}{$X^{2}=0.24, P=0.6$} \\
\hline & U & $21(12.1)$ & $38(13.5)$ & \\
\hline \multicolumn{2}{|l|}{ Consanguinity } & $110(44.4)$ & 116 (41.3) & $X^{2}=0.5, P=0.5$ \\
\hline \multicolumn{5}{|l|}{ Birth order } \\
\hline \multicolumn{2}{|l|}{ First } & $35(19.4)$ & $61(21.7)$ & \multirow[t]{5}{*}{$X^{2}=2.9, P=0.6$} \\
\hline \multicolumn{2}{|l|}{ Second } & $81(46.0)$ & $115(40.9)$ & \\
\hline \multicolumn{2}{|l|}{ Third } & $31(18.1)$ & 64 (22.9) & \\
\hline \multirow{2}{*}{\multicolumn{2}{|c|}{$\begin{array}{l}\text { Fourth } \\
\text { Fifth and more }\end{array}$}} & $25(13.7)$ & $33(11.7)$ & \\
\hline & & $5(2.8)$ & $8(2.8)$ & \\
\hline
\end{tabular}

Table 1: Socio-demographic data of cases vs. comparison children. 
Citation: $\quad$ Yahia S, El-Hadidy MA, El-Gilany AH, Mansour AK, Abdelmabood S, et al. (2015) Cognitive Function and Quality of Life in Egyptian Children with Acute Lymphoblastic Leukemia. J Blood Disord Transfus 6: 291. doi:10.4172/2155-9864.1000291

Page 3 of 4

\begin{tabular}{|l|l|l|l|}
\hline Quality of life & $\begin{array}{l}\text { Cases (177) } \\
\text { Median (Min-Max) }\end{array}$ & $\begin{array}{l}\text { Compared children (281) } \\
\text { Median (Min-Max) }\end{array}$ & Mann-Whitney test \\
\hline Health and activity problem & $450(0-800)$ & $800(678-800)$ & $Z=18.96, P \leq 0.001$ \\
\hline Emotion & $325(0-500)$ & $500(375-500)$ & $Z=18.3, P \leq 0.001$ \\
\hline Relation to others & $350(125-500)$ & $500(325-500)$ & $Z=17.9, P \leq 0.001$ \\
\hline School achievement & $200(0-500)$ & $500(250-500)$ & $Z=20.6, P \leq 0.001$ \\
\hline Total Quality of life scale & $1525(425-2250)$ & $2300(1925-2300)$ & $Z=20.9, P \leq 0.001$ \\
\hline
\end{tabular}

Table 2: Comparison between cases \& healthy children regarding pediatric quality of life inventory.

\begin{tabular}{|c|c|c|c|c|}
\hline \multicolumn{2}{|l|}{ Cognitive function tests } & \multirow{2}{*}{$\begin{array}{l}\text { Cases (177) } \\
\text { Median (Min-Max) } \\
9(2-10)\end{array}$} & \multirow{2}{*}{$\begin{array}{l}\text { Compared children (281) } \\
\text { Median (Min-Max) } \\
10(8-10)\end{array}$} & \multirow{2}{*}{$\begin{array}{l}\text { Mann-Whitney test } \\
Z=13.3, P \leq 0.001\end{array}$} \\
\hline Mini mental state & Orientation & & & \\
\hline & Registration & $3(2-3)$ & $3(2-3)$ & $Z=2.1, P=0.039$ \\
\hline & Attention & $5(2-5)$ & $5(4-5)$ & $Z=12.1, P \leq 0.001$ \\
\hline & Recall & $3(2-3)$ & $3(2-3)$ & $Z=5.01, P \leq 0.001$ \\
\hline & Language & $9(7-9)$ & $9(7-9)$ & $Z=9.3, P \leq 0.001$ \\
\hline & Total MMSE & $29(19-53)$ & $30(28-30)$ & $Z=14.1, P \leq 0.001$ \\
\hline \multirow[t]{2}{*}{ Tail making Test } & Tail-making A & $100(13-200)$ & $56(30-87)$ & $Z=14.7, P \leq 0.001$ \\
\hline & Tail-making B & $192(40-380)$ & $93(52-240)$ & $Z=12.2, P \leq 0.001$ \\
\hline
\end{tabular}

Table 3: Comparison between cases \& healthy children regarding cognitive function.

\section{Discussion}

Although a great attention has been paid to assess the adverse effect of cranial irradiation on the neurocognitive function for childhood leukemia survivors, much less efforts have been spent to evaluate similar effect caused by chemotherapy. In this study we tried to evaluate the cognitive function and quality of life for those children exposed to chemotherapy for leukemia treatment so that detecting those who are in need for psychiatric support or special education services in their academic career.

Major cognitive functions (orientation, registration, attention, recall, language and tail making tests) were significantly impaired in leukemic patients and survivors in the current study as compared to their age and sex matched controls. This is consistent with the results of Lofstad et al. [14] who evaluated cognitive functioning of 35 children and adolescents in long-term remission from ALL who were treated with central nervous system prophylactic chemotherapy only and found long-term squeal in global cognitive functions. This is explained by delayed brain changes, most commonly reduction in the white matter volume, intra cerebral calcifications especially with increased intensity of chemotherapy regimens including intrathecal (IT) methotrexate, and changes in glucose utilization and abnormalities in event related potential in those patients [15-18].

Moreover, Brown et al. [19] reported on 48 patients with moderaterisk ALL treated with a 3-year course of systemic and IT chemotherapy only and concluded that the participants had significantly poorer performance on tests of attention and memory as well as visual construction ability than those who had recently been diagnosed. Furthermore, these cognitive deficits tend to be not only persistent but also progressive throughout the adulthood decades after treatment from childhood ALL resulting in reduced educational attainment and unemployment $[20,21]$ making them at higher risk of suicidal ideation compared with siblings [22].

These results are to some extent different from those of Copeland et al. [23] who assessed the cognitive function of 99 children with different types of

cancer, with $73 \%$ of them being leukemia and lymphoma through neuropsychological tests and concluded that chemotherapy had only a slight effect on neurocognitive status and was confined to perceptual motor skills. This difference may be attributed to diversity of cancers in nature with different treatment protocols.

In addition, quality of life (QOL) in this study among childhood leukemia survivors was significantly lower as compared to age and sex matched healthy children. This is matched with Hicks et al. [24] who evaluated QOL for 13 children with ALL through phenomenological qualitative study and they concluded that QOL among ALL children was certainly affected and the most influencing factor attenuating their QOL was fatigue because of its adverse effect on their activity. This group of children usually face much more mental and emotional stress during their unpleasant visits to the clinics and hospitals, which makes them more vulnerable to fatigue [25]. Moreover, their school performance is adversely affected by poor social adjustment, including 
problems with peer relations, social withdrawal, and reduced social skills [26].

Furthermore, Zeltzer et al. [27] compared ALL survivors with healthy subjects. Survivors had lower physical, emotional, and vitality functions than normal population.

In contrast to the results in this study, other studies reported no difference or even higher scores in the group of childhood cancer survivors as compared to their healthy peers [28-30]. The possible explanation of the latter results is the desire of the survivors to be "as normal as possible," causing a response shift or "paradox of satisfaction" [31]. Moreover, childhood cancer survivors sometimes deny difficulties on QOL measures and to report high QOL even under difficult living conditions [32].

In conclusion we found that both neurocognitive function and QOL in leukemic children treated with chemotherapy alone without using craniospinal irradiation were significantly impaired as compared with healthy controls. So screening of intellectual and QOL aspects for those patients is mandatory so as to select those who have problems in achieving intellectual tasks or in leading normal lives and offer psychological and educational supports for them.

\section{References}

1. Compas BE, Jaser SS, Dunn MJ, Rodriguez EM (2012) Coping with chronic illness in childhood and adolescence. Annu Rev Clin Psychol 8: 455-480.

2. Askins M A, Moore B D (2008) Preventing Neurocognitive Late Effects in Childhood Cancer Survivors. J Child Neurol 23: 1160-1171.

3. Jansen NC, Kingma A, Schuitema A, Bouma A, Veerman AJ, et al. (2008) Neuropsychological outcome in chemotherapy-only-treated children with acute lymphoblastic leukemia. J Clin Oncol 26: 3025-3030.

4. Steen RG, Koury M, Granja CI, Xiong X, Wu S, et al. (2001) Effect of ionizing radiation on the human brain: White matter and gray matter $\mathrm{T} 1$ in pediatric brain tumor patients treated with conformal radiation therapy. Int J Radiat Oncol Biol Phys 49: 79-91.

5. Mulhern RK, Palmer SL, Reddick WE (2000) Quantitative white matter loss explains risks of young age for neurocognitive deficits in medulloblastoma survivors. Arch Clin Neuropsychol 15: 791-792.

6. Mulhern RK, Palmer SL, Reddick WE, Glass JO, Kun LE, et al. (2001) Risks of young age for selected neurocognitive deficits in medulloblastoma are associated with white matter loss. J Clin Oncol 19: 472-479.

7. Sheehan DV, Lecrubier Y, Sheehan KH, Amorim P, Janavs J, et al. (1998) The validity of Mini International Neuropsychiatric Interview (MINI). The development and validation of a structured diagnostic interview for DSM-IV and ICD-10. Journal of Clinical Psychiatry 59: 211-232.

8. Ibrahim M, Bishry Z, Hamed A (2002) Comparison of Mini International Neuropsychiatric Interview for children(MINI-KID) with the schedules for affective disorders and schizophrenia for school aged children ,present and lifetime version(KSADS-PL):In Egyptian sample presenting with childhood disorders. MD thesis, Ain Shams University.

9. Ouvrier RA, Goldsmith RF, Ouvrier S, Williams IC (1993) The value of the Mini-Mental State Examination in childhood: a preliminary study. J Child Neurol 8: 145-148.

10. Al-Rajeh S, Ogunniyi A, Awada A, Daif A, Zaidan R (1999) Preliminary assessment of an Arabic version of the Mini-Mental state examination. Ann Saudi Med 19: 150-152.

11. Rosen VM, Sunderland T, Levy J, Harwell A, McGee L, et al. (2005) Apolipoprotein $\mathrm{E}$ and category fluency: evidence for reduced semantic access in healthy normal controls at risk for developing Alzheimer's disease. Neuropsychologia 43: 647-658.
12. Elwan O, Hassan AA, Abdel Naseer M, Elwan F, Deif R, et al. (1997) Brain aging in a sample of normal Egyptians cognition, education, addiction and smoking. J Neurol Sci 148: 79-86.

13. Varni JW, Seid M, Rode CA (1999) The PedsQL: measurement model for the pediatric quality of life inventory. Med Care 37: 126-139.

14. Lofstad GE, Reinfjell T, Hestad K, Diseth TH (2009) Cognitive outcome in children and adolescents treated for acute lymphoblastic leukaemia with chemotherapy only. Acta Paediatr 98: 180-186.

15. Cole PD, Kamen BA (2006) Delayed neurotoxicity associated with therapy for children with acute lymphoblastic leukemia. Ment Retard Dev Disabil Res Rev 12: 174-183.

16. Reddick WE, Shan ZY, Glass JO, Helton S, Xiong X, et al. (2006) Smaller white-matter volumes are associated with larger deficits in attention and learning among long-term survivors of acute lymphoblastic leukemia. Cancer 106: 941-949.

17. Lesnik PG, Ciesielski KT, Hart BL, Benzel EC, Sanders JA (1998) Evidence for cerebellar-frontal subsystem changes in children treated with intrathecal chemotherapy for leukemia: enhanced data analysis using an effect size model. Arch Neurol 55: 1561-1568.

18. Zeller B, Tamnes CK, Kanellopoulos A, Amlien IK, Andersson S, et al. (2013) Reduced neuroanatomic volumes in long-term survivors of childhood acute lymphoblastic leukemia. J Clin Oncol 31: 2078-2085.

19. Brown RT, Madan-Swain A, Pais R, Lambert RG, Sexson S, et al. (1992) Chemotherapy for acute lymphocytic leukemia: cognitive and academic sequelae. J Pediatr 121: 885-889.

20. Krull KR, Brinkman TM, Li C, Armstrong GT, Ness KK, et al. (2013) Neurocognitive outcomes decades after treatment for childhood acute lymphoblastic leukemia: a report from the St Jude lifetime cohort study. J Clin Oncol 31: 4407-4415.

21. Armstrong GT, Reddick WE, Petersen RC, Santucci A, Zhang N, et al. (2013) Evaluation of memory impairment in aging adult survivors of childhood acute lymphoblastic leukemia treated with cranial radiotherapy. J Natl Cancer Inst 105: 899-907.

22. Brinkman TM, Zhang N, Recklitis CJ, Kimberg C, Zeltzer LK, et al. (2014) Suicide ideation and associated mortality in adult survivors of childhood cancer. Cancer 120: 271-277.

23. Copeland DR, Moore BD 3rd, Francis DJ, Jaffe N, Culbert SJ (1996) Neuropsychologic effects of chemotherapy on children with cancer: a longitudinal study. J Clin Oncol 14: 2826-2835.

24. Hicks J, Bartholomew J, Ward-Smith P, Hutto CJ (2003) Quality of life among childhood leukemia patients. J Pediatr Oncol Nurs 20: 192-200.

25. Davies B, Whitsett SF, Bruce A, McCarthy P (2002) A typology of fatigue in children with cancer. J Pediatr Oncol Nurs 19: 12-21.

26. Moyer KH, Willard VW, Gross AM, Netson KL, Ashford JM, et al. (2012) The impact of attention on social functioning in survivors of pediatric acute lymphoblastic leukemia and brain tumors. Pediatr Blood Cancer 59: 1290-1295.

27. Zeltzer LK, Lu Q, Leisenring W, Tsao JC, Recklitis C, et al. (2008) Psychosocial outcomes and health-related quality of life in adult childhood cancer survivors: a report from the childhood cancer survivor study. Cancer Epidemiol Biomarkers Prev 17: 435-446.

28. Zebrack BJ, Chesler MA (2002) Quality of life in childhood cancer survivors. Psychooncology 11: 132-141.

29. Maunsell E, Pogany L, Barrera M, Shaw AK, Speechley KN (2006) Quality of life among long-term adolescent and adult survivors of childhood cancer. J Clin Oncol 24: 2527-2535.

30. Harila MJ, Salo J, Lanning M, Vilkkumaa I, Harila-Saari AH (2010) High health-related quality of life among long-term survivors of childhood acute lymphoblastic leukemia. Pediatr Blood Cancer 55: 331-336.

31. Sprangers MA, Schwartz CE (1999) The challenge of response shift for quality-of-life-based clinical oncology research. Ann Oncol 10: 747-749.

32. O'Leary TE, Diller L, Recklitis CJ (2007) The effects of response bias on self-reported quality of life among childhood cancer survivors. Qual Life Res 16: 1211-1220. 